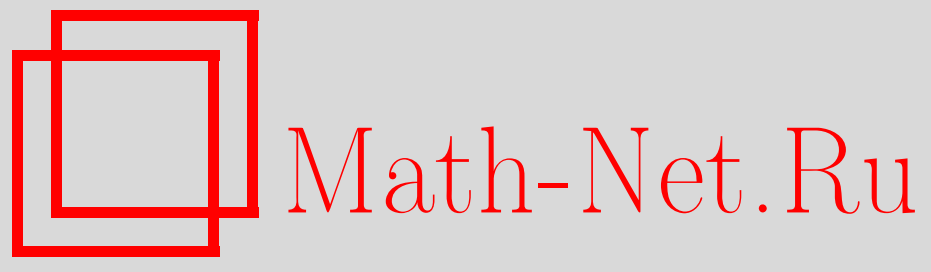

О. В. Герасимова, Спектр коммутаторного гамильтониана сродни энергетическим уровням атома водорода, УМH, 2009, том 64, выпуск 4, 177-178

DOI: https://doi.org/10.4213/rm9293

Использование Общероссийского математического портала Math-Net.Ru подразумевает, что вы прочитали и согласны с пользовательским соглашением http: //www. mathnet.ru/rus/agreement

Параметры загрузки:

IP: 3.85 .183 .62

26 апреля 2023 г., 12:00:25

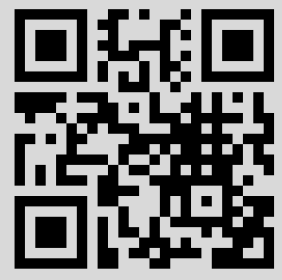




\section{Спектр коммутаторного гамильтониана сродни энергетическим уровням атома водорода}

\section{О. В. Герасимова}

Tеорема. Пусть мера Хаара $\mu$ на компактной группе $G$ такова, что $\mu(G)=1$, а $\rho: G \rightarrow \operatorname{End}_{K} V-$ непрерывное представление $G$ в банаховом пространстве $V$ над полем комплексных чисел $K$ и линейный оператор $H_{[G, G]}^{\rho}$ определяется формулой

$$
H_{[G, G]}^{\rho} \stackrel{\text { def }}{=} \iint_{G} \rho([g, h]) d \mu_{g} d \mu_{h} \quad\left([g, h] \stackrel{\text { def }}{=} g^{-1} h^{-1} g h\right) .
$$

Тогда для любого п-мерного подпространства $W$, на котором $\rho(G)$ действует неприводимо,

$$
H_{[G, G]}^{\rho}(w)=\frac{1}{n^{2}} \cdot w \quad(w \in W) .
$$

ВАжный ПРимеР. Пусть $\mathrm{SU}(2, K)$ - группа унитарных $(2 \times 2)$ матриц над комплексным полем $K$ с детерминантом, равным единице, $V$ - линейное пространство всех функций $f: G \rightarrow K$, для которых $\|f\|^{2} \stackrel{\text { def }}{=} \int_{G}|f(g)|^{2} d \mu_{g}<\infty$, и представление $\rho: G \rightarrow \operatorname{End}_{K} V$ группы $G$ реализуется правыми сдвигами $\rho(h) f(g) \stackrel{\text { def }}{=} f(g h)$. Хорошо известно (см. [1]-[3]), что:

а) в $V$ с точностью до эквивалентности функций реализуется гильбертово пространство относительно полуторалинейной формы $(f(g), h(g)) \stackrel{\text { def }}{=} \int_{G} f(g) \overline{h(g)} d \mu_{g}$;

б) для любого натурального $n$ единственное с точностью до изоморфизма $n$-мерное представление группы $G$ реализуется в $V$ и имеет в $V$ кратность, равную $n$;

в) сумма всех конечномерных неприводимых подпространств из $V$ плотна в $V$.

Из теоремы заключаем, что в гильбертовом пространстве $V$ имеется полный ортонормированный базис, состоящий из собственных векторов оператора $H_{[G, G]}^{\rho}$. В частности, для любого натурального $n$ кратность собственного значения $1 / n^{2}$ равна $n^{2}$. (См. для сравнения в книге [4] спектр оператора Шрёдингера для атома водорода.)

ПреДЛОЖенИЕ. Для любого непрерывного представления $\rho: G \rightarrow \operatorname{End}_{K} V$ компактной группы $G$ в банаховом пространстве $V$ над полем комплексных чисел $K$ $u$ любых $t, h \in G$ в пространстве непрерывных операторов $E(V)$ имеет место равенство

$$
\int_{G} \rho\left(g^{-1}\right) * \rho\left(t^{-1} * h * t\right) * \rho(g) d \mu_{g}=\int_{G} \rho\left(g^{-1}\right) * \rho(h) * \rho(g) d \mu_{g} .
$$

Обозначим через $\chi_{\rho}(h)$ правую часть этого равенства и продолжим по линейности отображения $\rho: G \rightarrow E(V), \chi_{\rho}: G \rightarrow E(V)$ на любые формальные конечные линейные комбинации групповой алгебры $K[G]$. Тогда $\chi_{\rho}\left(a_{1} * a_{2}\right)=\chi_{\rho}\left(a_{2} * a_{1}\right)$ для всех $a_{1}, a_{2} \in K[G]$. Ограничим операторы $\chi_{\rho}(K[G])$ на любое конечномерное инвариантное подпространство $W$, не содержащее нетривиальных инвариантных подпространств относительно действия $K[G]$. Тогда в силу непрерывности $\rho$ и того, что интеграл является пределом конечных частичных сумм, имеем

$\left.\chi_{\rho}(h)\right|_{W}=\left.\left.\left.\int_{G} \rho\left(g^{-1}\right)\right|_{W} * \rho(h)\right|_{W} * \rho(g)\right|_{W} d \mu_{g}=\int_{G} \rho_{W}\left(g^{-1}\right) * \rho_{W}(h) * \rho_{W}(g) d \mu_{g}=\chi_{\rho_{W}}(h)$,

где непрерывное представление $\rho_{W}: G \rightarrow \operatorname{End}_{K} W$ группы $G$ в банаховом подпространстве $W$ таково, что $\left.\rho_{W}(h) \stackrel{\text { def }}{=} \rho(h)\right|_{W}$ и $\chi_{\rho_{W}}\left(a_{1} * a_{2}\right)=\chi_{\rho_{W}}\left(a_{2} * a_{1}\right)$. Определим 
отображение $\overline{\chi_{\rho_{W}}}: \operatorname{End}_{K} W \rightarrow \operatorname{End}_{K} W$, полагая для любого $a \in \operatorname{End}_{K} W$

$$
\overline{\chi_{\rho_{W}}}(a) \stackrel{\text { def }}{=} \int_{G} \rho_{W}\left(g^{-1}\right) * a * \rho_{W}(g) d \mu_{g} .
$$

СлеДСтвиЕ 1. Для любого элемента $a \in \operatorname{End}_{K} W$

$$
\overline{\chi_{\rho_{W}}}(a)=\frac{1}{n} \operatorname{tr}(a) 1_{W} \quad\left(n=\operatorname{dim}_{K} W, 1_{W}-\text { единица в } \operatorname{End}_{K} W\right) .
$$

Доказательство. Ясно, что $\chi_{\rho_{W}}(d)=\overline{\chi_{\rho_{W}}}\left(\rho_{W}(d)\right)$. Так как $\rho_{W}: K[G] \rightarrow \operatorname{End}_{K} W$ является неприводимым представлением в конечномерном пространстве $W$ и поле $K$ алгебраически замкнуто, то по теореме плотности будем иметь $\rho_{W}(K[G])=\operatorname{End}_{K} W$ и $\overline{\chi_{\rho_{W}}}\left(d_{1} * d_{2}\right)=\overline{\chi_{\rho_{W}}}\left(d_{2} * d_{1}\right)$. Хорошо известно, что в конечномерном случае линейное подпространство линейных операторов с нулевым следом в $\operatorname{End}_{K} W$ порождается коммутаторами $a_{1} * a_{2}-a_{2} * a_{1}\left(a_{1}, a_{2}\right.$ пробегают $\left.\operatorname{End}_{K} W\right)$. Но $\overline{\chi_{\rho_{W}}}$ на этом подпространстве коразмерности 1 принимает нулевое значение. Следовательно, образ $\overline{\chi_{\rho_{W}}}$ должен лежать в каком-то одномерном подпространстве. Прямое вычисление дает

$$
\overline{\chi_{\rho_{W}}}\left(1_{W}\right)=\int_{G}\left(\rho_{W}\left((g)^{-1}\right) * 1_{W} * \rho_{W}(g)\right) d \mu_{g}=\int_{G} 1_{W} d \mu_{g}=1_{W} .
$$

Следовательно, обе части (1) принимают на $\operatorname{End}_{K} W$ одинаковые значения.

Следствие 2. Для произвольного базиса матричных единии $\left\{E_{i j} \mid i, j=1, \ldots\right.$, $\left.n=\operatorname{dim}_{K} W\right\}$ и любого $a \in \operatorname{End}_{K} W$

$$
\overline{\chi_{\rho_{W}}}(a)=\frac{1}{n} \sum_{i=1}^{n} \sum_{j=1}^{n} E_{i j} * a * E_{j i} .
$$

Но тогда, используя (2), получаем цепочку равенств

$$
\begin{aligned}
\left(\int_{G} \rho\right. & \left.\left(g^{-1}\right)\left(\int_{G} \rho\left(h^{-1}\right) \rho(g) \rho(h) d \mu_{h}\right) d \mu_{g}\right) \times w \\
& =\left(\int_{G} \rho_{W}\left(g^{-1}\right)\left(\frac{1}{n} \sum_{i=1}^{n} \sum_{j=1}^{n} E_{i j} * \rho_{W}(g) * E_{j i}\right) d \mu_{g}\right) \times w \\
& =\left(\frac{1}{n} \sum_{i=1}^{n} \sum_{j=1}^{n}\left(\int_{G} \rho_{W}\left(g^{-1}\right) * E_{i j} * \rho_{W}(g) d \mu_{g}\right) * E_{j i}\right) \times w \\
& =\left(\frac{1}{n} \sum_{i=1}^{n} \sum_{j=1}^{n}\left(\frac{1}{n} \operatorname{tr}\left(E_{i j}\right) 1_{W}\right) * E_{j i}\right) \times w=\frac{1}{n^{2}} \cdot 1_{W} \times w=\frac{1}{n^{2}} \cdot w .
\end{aligned}
$$

Эти аргументы справедливы и при другом порядке интегрирования и приводят к тому же ответу. Теорема полностью доказана.

\section{Список литературы}

[1] Л. С. Понтрягин, Непреръвнъе группъ, 4-е изд., Наука, М., 1984. [2] Д. П. Желобенко, Компактные группы Ли и их представления, Наука, М., 1970; англ. пер.: D. Р. Želobenko, Compact Lie groups and their representations, Transl. Math. Monogr., 40, Amer. Math. Soc., Providence, RI, 1973. [3] М. А. Наймарк, Нормированные колъца, 2-е изд., Наука, М., 1968; англ. пер. 1-го изд.: М. А. Naimark, Normed rings, Noordhoff, Groningen, 1964. [4] Г. Вейль, Теория групп и квантовая механика, Наука, М., 1974; пер. с нем.: H. Weyl, Gruppentheorie und Quantenmechanik, Hirzel, Leipzig, 1931.

О. В. Герасимова (O. V. Gerasimova)

Московский государственный университет им. М. В. Ломоносова

E-mail: ynona_olga@rambler.ru
Представлено А. В. Михалёвым Принято редколлегией 14.04.2009 